\title{
Well-being Supportive Home Environment of Elderly People with Visual Impairments and Health-related Quality of Life
}

The aim of this study was to describe the well-being supportive home environment of elderly people ( $\mathrm{n}=37$ ) with visual impairment $(\mathrm{VI})$ and its relationship with health-related quality of life (HRQoL). Data were collected during home visits six (6) months after the commencement of individual low vision rehabilitation (LVR) process. A structured well-being supportive environment instrument and a general HRQoL instrument were administered. Background variables had no detectable effect on the HRQoL. The HRQoL correlated significantly with the symbolic environment living-related fears and feelings of general restrictiveness. The participants generally saw their home environment to be comfortable and supportive of well-being, but many felt that their life was too much restricted to home environment.

Keywords: home environment; elderly people; visual impairment; health-related quality of life, $15 \mathrm{D}$ 


\section{Introduction}

The $21^{\text {st }}$ century will have a silver lining as it will be defined by global aging (International Federation on Aging [IFA]); one fifth of the population of Europe was aged 65 or above in 2017 (Eurostat, 2018). Consequently, policies, actions, strategies and projects designed to promote healthy, independent, and active aging are being implemented (Positive Aging, 2016; PRO HEALTH 65+; United Nations Economic Commission for Europe [UNECE], 2012; World Health Organization [WHO], 2012, 2015) to address the new challenges that aging populations present to existing health and welfare systems (Rechel et al., 2013). Aging well at home and in community settings is a major theme in European Aging Research (Futurage, 2011), and the creation of more agefriendly and well-being supportive environments has become a key objective for various social actors (WHO, 2018). According to Nygren et al. (2007), very old European people living alone in urban areas share a common home experience despite cultural differences and individual variability.

The subjective well-being of elderly people warrants attention because it may play a protective role and help maintain health and quality of life while aging (Steptoe et al., 2015). To achieve the objective that elderly people should "age in place", it is necessary to recognise that environmental characteristics strongly affect perceived quality of life (QoL) in old age (Schorr and Khalaila, 2018). In particular, it is essential to recognize the home as an environment in which healthcare is designed and provided (Barry et al., 2018). A person's environment has an enormous impact on how and to what extent they experience disability 
(WHO, 2011). Good-quality environments (including home and neighbourhood environments) support active and healthy aging. In this context, a good quality environment is one with good pavements, cycling paths, public transportation, and safe crossings as well as readily accessible nearby primary healthcare centres, shops, libraries, and green spaces (Rechel et al., 2013). Accessible habitation increases the perceived usefulness and meaningfulness of homes while reducing dependence on external help with routines and everyday activities related to housing (Nygren et al., 2007). Previous studies have shown that easy access to services and sites in the living area has a positive effect on quality of life (QoL) in old age, and the same is true for the ability to get around (Schorr \& Khalaila, 2018; LaGrow et al., 2011).

Healthy life years have increased over the last few decades, but mild disability has also become more common (Rechel et al., 2013). Visual and hearing impairment are the two leading causes of old-age disability (WHO 2011). Vision loss is not an inevitable part of aging and many avoid vision problems (IFA). Nevertheless, the incidence of chronic eye diseases increases with age. The global population is aging and these problems are increasing concurrently (WHO, 2013). It is estimated that there are 253 million visually impaired (VI) people in the world today, and most of them are over 50 years of age (WHO, 2013; Bourne et al., 2017).

The definition of visual impairment (VI) includes both moderate and severe visual impairment as well as blindness (WHO, 2012). WHO categories of VI are based on the best corrected visual acuity (BCVA) of the better eye, and the visual 
field. Categories 1-2 represent moderate and severe VI, while categories 3-5 represent blindness. WHO category 1 includes individuals with BCVA below 0.3 or $6 / 18$ but at least 0.1 or $6 / 60$. Category 2 includes individuals with BCVA below 0.1 or $6 / 60$, but at least 0.05 or $3 / 60$. WHO category 3 (deep visual impairment) includes individuals with BCVA below 0.05 or $3 / 60$, but at least 0.02 or 2/100 or with a $<10$ degree radius of the visual field. Categories 4 and 5 include nearly blind and totally blind individuals. The eye disease most often responsible for VI in European and other developed countries is age-related macular degeneration (AMD), for which the worldwide pooled prevalence at age range $45-85$ is $8,01 \%$ (Wong et al., 2014). The number of people suffering from AMD is expected to rise from 196 million in 2020 to 288 million in 2040 (Wong et al., 2014). In addition, the prevalence of AMD after the age of 75 is increasing rapidly in Europe (Wong et al., 2014). AMD is estimated to affect roughly as many people as Alzheimer's disease (WHO, 2011).

Vision loss does not only affect the individuals suffering from it, but also their families and friends, communities, and nations. This cumulative snowball effect is often underestimated (IFA). Elderly people with VI are doubly burdened; both loss of vision and the general consequences of aging restrict their participation in society (Alma et al., 2011). VI challenges independent living and the ability to perform daily tasks. Older adults with VI have indeed been found more dependent in both personal and instrumental daily life activities - such as cutting their toenails, cooking, cleaning, reading, going out in the neighbourhood (Ivanoff et al., 2000) - than healthy controls. Moreover, they have been shown to participate less in society, which is essential for active aging, well-being, and 
quality of life (Alma et al., 2011). Low vision rehabilitation (LVR) is a multidisciplinary professional service that aims at optimal use of residual visual functions and re-training of skills, and also re-integration in society (Markowitz, 2016). Consequently, there is a clear need for further research on public health needs related to eye health (WHO, 2013) as well as actions to help visually impaired people (Markowitz, 2016).

Previous studies have examined outdoor mobility in old age by looking at the associations between barriers in the outdoor environment and perceived quality of life (Rantakokko et al., 2010), but provide no insight into how elderly people with VI describe and perceive their home environment or the relationship between these perceptions and health-related quality of life (HRQoL). Additionally, the systematic review of Binns et al. (2012) concluded that there is an urgent need for research on LVR and QoL related to the home environment and home-based rehabilitation services. Previous studies have demonstrated an increased risk of falls as well as fear and perceived difficulties of moving outdoors among elderly people with VI (Rokicki et al., 2016; Wood et al., 2011; Patino et al., 2010; Riazi et al., 2016), emphasizing the need to study elderly people's perceptions of their habitation as a key indicator of their health (Tomsone et al., 2013). The current study aims to fill this gap in knowledge related to aging in place and the perceptions of the home environment by those with VI.

In this study, the well-being supportive home environment is defined according to the 
theory of Elo et al. (2011) in terms of physical, social, and symbolic aspects as well as through the sense of security. The environmental well-being of elderly home-dwelling people with VI is analyzed using health-related quality of life (HRQoL), which is understood as a multidimensional concept (Sintonen, 2001) that encompasses physical, emotional, and social factors associated with disability. The aim of this study is to describe the well-being supportive home environment of elderly people with VI and its relationship with HRQoL. The knowledge that can be useful in planning interventions to improve the home environment of elderly individuals with VI and to develop low vision rehabilitation services to support independent living and active aging within this group. The research questions addressed in this study are: 1) How do elderly people with VI evaluate their home environment? 2) Is the perception of the well-being supportive home environment related to the HRQoL among elderly people with VI? 


\section{Materials and Methods}

The study is part of a prospective study of a patient cohort $(n=39)$ comprising consecutive home-dwelling elderly people aged 65 or above referred to the LVR services of Oulu University Hospital's Low Vision Centre (OLVC) because of VI over the course of one year (May 2016-May 2017). VI was defined according to the criteria of the World Health Organization (WHO 2003). Informed consent was given by all participants.

Data were collected during a home visit by the first author six (6) months after the commencement of each participant's LVR. The structured well-being supportive environment instrument (Elo et al., 2011) and the generic 15D health-related quality of life (HRQoL) instrument (Sintonen 2001) were included in the interviews. This approach was chosen because the participants were unable to self-administer the questionnaires due to VI. One participant withdrew her previously given consent, and another could not be reached to arrange the home visit, so 37 subjects were interviewed in total.

The well-being supportive environment instrument used in this study is based on an instrument development and theory testing process introduced by Elo (2006) to assess environments that support the well-being of elderly people in northern regions. The instrument originally consisted of 100 items. A shortened version of the instrument was used in this study. The abbreviated instrument has 30 
items: 9 relating to the physical environment, 12 to the social environment, and 9 to the symbolic environment. Each item is answered using a Likert-type scale with possible responses ranging from complete agreement to complete disagreement. The physical environment is evaluated in terms of safety at home, the pleasance of the physical environment, and the safety of the living environment. The social environment is analyzed in terms of the pleasance of the social environment, interpersonal relationships, and ability to get help. The symbolic environment is analyzed in terms of the respondent's fears, the natural environment (nature), the respondent's mood, and feelings of restrictiveness.

The 15D HRQoL instrument is comprehensive questionnaire covering 15 dimensions of health: mobility, vision, hearing, breathing, sleeping, eating, speech, excretion, usual activities, mental function, discomfort and symptoms, depression, distress, vitality, and sexual activity. For each item, the respondent chooses the ordinal level that best describes his/her health status with respect to the relevant dimension. Missing data due to unanswered questions were replaced using a combined valuation and replacement algorithm developed for the instrument (Sintonen, 2018). Individuals' responses to the 15D HRQoL instrument were used to generate respondent-specific index scores based on a set of utility or preference weights. These index scores take values from 0 to 1 , where 0 corresponds to being dead and 1 to the best possible health.

Data on respondents' year of birth, gender, marital status, habitation, type of 
accommodation, and use of services were also gathered as background variables. Data on the participants' ophthalmological status - specifically diagnoses related to VI and the degree of VI - dates 6 months back as it had been evaluated at the onset of the LVR process.

IBM SPSS $®$ (version 24.0, SPSS Inc., Chicago, IL, USA) was used to store and analyse the data. Data were characterized using descriptive statistics including frequencies and percentages for graded variables and minimum, maximum, mean, and standard deviation for continuous variables. Differences between variables were evaluated by cross-referencing and computing correlation coefficients. Sum variables were formed from the items describing the physical, social, and symbolic dimensions of the well-being supportive environment. Oneway ANOVA and independent samples t-test were used to measure differences between groups, with confidence intervals of $95 \%$ and a p-value threshold of < 0.05 for significance.

The study was approved by the Northern Ostrobothnia Hospital district and the Regional Ethics Committee (36/2016). The study was conducted in accordance with good scientific practice and the statements of the Helsinki Declaration (Finnish National Board on Research Integrity [TENK], 2012; World Medical Association [WMA], 2013). 


\section{Results}

The respondents' ages ranged from 71 to 94; most of them (59\%) were aged 80 to 89 years, and just over a fifth (22\%) had reached the age of 90 or above. The mean age was 84 years ( \pm SD 6.313). Twenty-six (70\%) were women. Seventeen (46\%) of the respondents were married and thirteen (35\%) were widows. Twenty-two (60\%) lived alone. Different types of residence were evenly represented: thirteen (35\%) lived in a detached house, twelve (32\%) in an apartment, and ten $(27 \%)$ in a row house. Two of the respondents lived in sheltered accommodation. The twenty-seven (73\%) used some services or received help to support their daily life, the most common being cleaning service (38\%) following by transport services (32\%) and security and home care services $(30 \%)$. Background information on the participants is presented in Table 1.

Retinal diseases including AMD, proliferative diabetic retinopathy, diabetic maculopathy, macular hole, and central retinal venous occlusion were the most common diagnoses of primary eye disease, and were responsible for VI in most cases $(n=31)$. The remaining VIs were due to glaucoma $(n=5)$ or corneal disease $(n=1)$. According to the WHO classification, most $(n=32)$ of the participants were moderately visually impaired, a few (4) were severely visually impaired, and one was deeply visually impaired.

On average, the responses reflected a moderate HRQoL. The mean 15D HRQoL index 
score for the participants was $0.758( \pm$ SD 0.086$)$, but there were substantial individual differences; the worst individual 15D HRQoL index score was 0.609 and the best 0.927 . Interestingly, the severity of VI was not significantly related to the 15D HRQoL index score $(p=0.176)$. The respondents experienced the greatest difficulties in the dimension of seeing (mean 0.360) with lesser difficulties in the dimensions of usual activities (0.600), sexual activity (0.605), and moving (0.665). The respondents experienced the least difficulties in the dimensions of eating (0.962) and speech (0.952). The age groups with the highest (0.790) and lowest (0.71) mean 15D scores were 75-79 years and 70-74 years, respectively. However, there was no significant relationship ( $p=0.683$ ) or correlation $(\mathrm{r}=0.079)$ between the age and the HRQoL. Unmarried and single subjects had a lower mean $15 \mathrm{D}$ score $(0.712)$ than married or cohabiting subjects $(0.785, p=0.470)$. However, people living alone had a slightly higher HRQoL (0.762) than those living with someone else $(0.752, p=0.736)$. These betweengroup differences were not statistically significant $(p=0.470$ and $p=0.736$, respectively). Subjects living in sheltered homes did not have significantly $(\mathrm{p}=0.509)$ better HRQoL scores than those living in apartments. Having a garden to care for appeared to be associated with a higher HRQoL $(p=0.050)$. The relationships between the mean 15D scores and the studied background variables are presented in Table 1.

A clear majority (33/89\%) of participants found their homes to be comfortable and their physical $(28 / 76 \%)$ and social $(24 / 65 \%)$ environments to be pleasant, but well over half $(24 / 65 \%)$ somewhat or fully disagreed with the statement that they have no problems moving around outside their home, and twenty-three (62\%) 
somewhat or fully agreed that their lives were too heavily restricted to their home environment. One in three (11/30\%) felt that there were not enough services available where they lived, and a quarter (9/25\%) felt that they could not move safely in their living environment. The vast majority $(30 / 81 \%)$ of the respondents did not experience a need for improvements in their home, but a quarter $(9 / 25 \%)$ felt that they had insufficient lighting in their home to support their daily activities during both day and night. Over one quarter (10/27\%) reported having insufficient contact with friends and relatives, and around a quarter $(9 / 24 \%)$ found their peer support inadequate. Half $(19 / 51 \%)$ of the respondents felt that changes in their physical condition had limited their social participation. Descriptive statistics for items relating to the well-being supportive home environment of the elderly participants and their correlations to 15D HRQoL index scores are presented in Table 2.

The correlations between the 15D HRQoL score and the sum variables relating to the physical $(r=0.310)$ and social environment $(r=0.221)$ were positive, but not statistically significant $(\mathrm{p}=0.066$ and $\mathrm{p}=0.196$, respectively). However, the need for improvements to home environment to manage better there correlated statistically significantly with the $15 \mathrm{D}$ HRQoL score $(\mathrm{r}=-0.365, \mathrm{p}=0.028)$, as did safe mobility in the living area $(r=0.346(\mathrm{p}=0.039)$ and having no problems moving outside the home $(\mathrm{r}=0.411, \mathrm{p}=0.013)$.

The 15D HRQoL scores correlated statistically significantly with the symbolic environment sum variable $(r=-0.607, \mathrm{p}<0.001)$. Dimensions of the symbolic environment include fears related to living, the natural environment and mood, 
feelings of social restrictiveness, and feelings of general restrictiveness related to functioning and disability. Living-related fears $(r=-0.655, \mathrm{p}=<0.001)$ and feelings of general restrictiveness related to functioning and disability $(\mathrm{r}=\mathrm{-}$ $0.536, \mathrm{p}=0.001)$ were statistically significantly related to the HRQoL score. Perceived HRQoL deteriorated with increasing living-related fears and general feelings of restrictiveness related to functioning and disability. A detailed analysis of these items revealed that participants who somewhat or fully agreed that their weakened physical condition considerably restricted their life perceived their HRQoL to be significantly worse than those who somewhat or fully disagreed with this item $(\mathrm{p}=0.004)$. Similarly, those who felt that the treatment of their illnesses restricted their life considerably perceived their HRQoL to be worse than those who did not feel so restricted $(p=0.033)$. Those who were afraid of falling or other accidents at home $(\mathrm{p}=0.011)$ or moving outdoors alone $(\mathrm{p}=0.001)$ scored worse than those who somewhat or fully disagreed with these items. 


\section{Discussion}

This study was conducted to assess the relationship between HRQoL among elderly people with VI and their perceptions of the well-being supportive environment. The elderly respondents generally perceived their home environments to be comfortable and well-being supportive, but they also perceived their lives to be restricted to the home environment to some degree, and they considered their VI to have reduced their ability to maintain social relationships. In addition, outdoor mobility and running errands were perceived to be challenging.

The "feelings of restrictiveness" dimension considered in this study cannot be interpreted as being "stuck in place" in the manner described by Leibing et al. (2016), who use it in reference to a situation where a person with declining health is confronted with the need to consider moving to another living space to survive better. An earlier study (Macnaughton et al., 2019) concluded that people with VI only experience difficulties in mobility indoors if the environment is unfamiliar. However, perceived difficulties with mobility outdoors, hobbies, shopping, dining out, and using public transportation (Macnaughton et al., 2019) burden the visually impaired because they contribute to social isolation. Slowly, the threshold of the home increases and the disability causes the individual's life to be excessively restricted to the home environment. Previous research has highlighted the ability to engage in various hobbies and meaningful activities as a significant contributor to well-being among elderly people (Elo et al., 2011). 
It has been suggested that problems with accessibility increase feelings of insecurity about going outside. This further reduces social interaction and activity in the form of informal meetings, e.g. in shops and parks. (Schorr \& Khalaila, 2018). This study supports previous findings indicating that inability to access services and move safely in the living area are clinically significant, and that encumbering features such as perceived barriers in the outdoor environment adversely affect the QoL of elderly people. Fear of moving outdoors and unmet needs for physical activity mediate this association (Rantakokko et al., 2010).

It is also notable that according to Elo et al. (2011), the northern environment poses unique challenges to elderly people's outdoor mobility and ability to cope with everyday life. Snowy and icy winter conditions make it difficult for people with VI to leave home, particularly if needing to do so alone. Darkness and poor weather conditions restrict social interaction (Elo et al., 2011), snow and ice make footpaths slippery, and snow may dazzle in bright weather, lowering contrasts and endangering orienteering. A third of the respondents somewhat or fully disagreed that sidewalks and cycling paths in their neighborhood were kept clean and safe during wintertime. Therefore, the areas they inhabited were not perceived as being safe to use year round.

There were no statistically significant differences in HRQoL between different age groups, between respondents of different marital status, or between respondents living in different types of accommodation. However, having a garden to care for correlated significantly and positively with the HRQoL. This is consistent with previous findings that gardening promotes overall health and quality of life 
(Wang and MacMillan 2013). There is evidence that marriage or cohabitation also improve the HRQoL (Schorr \& Khalaila, 2018). This could not be confirmed by the current study; statistically significant relationship was not found in the small sample though there were a trend in the direction.

The impact of perceived problems related to moving outdoors and fear of falls or accidents at home and outdoors is supported by previous studies (Macnaughton et al., 2019). People with VI perceive mobility, both in- and outdoors, as a key rehabilitation need, so it should be addressed early in the LVR process (Macnaughton et al., 2019). Brundle et al. (2015) note that it is not ultimately the environment that causes falls but how a person interacts with it, and therefore argue that environmental modifications should be accompanied by behavioral assessments. Behavioral assessments for elderly people with VI could include mobility training and the use of aids to encourage proactivity and independence when moving outdoors even though it might feel frightening. Some individuals may also require external help in moving outdoors and running errands. Agencies that could provide external help can be for example friends, relatives, peers, volunteers, home care staff, or a personal assistant.

Bright light is universally considered helpful for people with VI, but there are individual differences and preferences regarding lighting levels among VI people (Riazi et al., 2012). Lewis and Torrington (2013) note that daylight and sunlight are especially important for homebound elderly people with VI because they can support a sense of connection to the outside world. Many of the elderly participants in this study felt that they had insufficient lighting, but most of them 
did not report a pressing need for improvements in their home. This may be due to a lack of knowledge about the home lighting adjustments that are available, as concluded by Riazi et al. (2012) in their study on assistive devices and home modifications using a similar study population and setting. In Finland, individuals with a certain degree of VI can get socially funded upgrades to their home lighting.

The sample was drawn from one of Finland's five university hospital districts, and it included individuals from both rural and urban environments. Notably, most of the participants had other diseases and health issues in addition to VI, which may have affected their well-being and perceived HRQoL. These issues were not recorded in this study. We would like to emphasize, that the study sample is representative of the Finnish registered VI population (Ojamo, 2017). The respondents were relatively old, reflecting the general demographics of aged individuals with VI in Finland (Ojamo, 2017) and other western countries (Bourne et al., 2017). The sample was also representative of the international population with VI (Bourne et al., 2017) because most of the respondents were women and the degree of VI was typically moderate. The instruments used in this study are valid and reliable for assessing environmental well-being and the HRQoL of elderly people in northern environments. The data were collected over the course of one year, at six months after the commencement of the participant's LVR in each case, and all four seasons were covered. Participants interviewed during the winter months may have raised the problem of slippery footpaths and snow-crashing more frequently than those interviewed during the summer. 
The well-being supportive environment instrument has been validated by expert and panel evaluation, and by both principal component and confirmatory factor analyses (Elo 2006, Elo et al., 2011, 2013). It was designed to assess the perceptions of elderly people living in northern regions. The $15 \mathrm{D}$ instrument is recommended by the Washington Panel and it is available in numerous languages. The validity and reliability are considered to be high. (Sintonen, 2001). It has been used in several studies in Finland and other countries to investigate the HRQoL of various patient groups in relation to diverse health problems and diagnoses (Sintonen, 2018).

LVR can encompass a wide range of different interventions. Appropriate accessibility modifications in homes can meet the needs of the people with functional limitations and impairments that threaten their survival in the home environment (Cho et al., 2016). However, the results of this study suggest that interventions are also needed at the neighborhood level to ensure the safety of elderly people with VI, and that such interventions can support their well-being, survival at home, active aging, and aging in place. These findings could be used to improve LVR processes by addressing key environmental issues and related factors as perceived by elderly people. They also provide a basis for evaluating the effectiveness of LVR by planning and implementing intervention studies. There is a clear need for a robust evidence base to support LVR services and ensure they can meet future needs. Future interventions and research on the home environments of elderly people with VI should pay more attention to the social 
environment and psychological factors relating to fears of moving rather than concentrating only on physical aspects and obstacles in the home environment.

Interventions should aim at promoting active aging, independent functioning and participation in modern digital society through various technologies where both, in-home and outdoor environments are addressed with holistic approach. Technology holds the key for future LVR as it can facilitate numerous everyday tasks, such as reading, writing, communication, navigation and information retrieval (Markowitz, 2016), and alleviate social isolation and loneliness (Khosravi et al., 2016). An effective consortium to provide such interventions could be formed by a mix healthcare professionals, third sector actors and technology experts not to forget about social work professionals, occupational therapists and experts in the field of education. Education and training is needed to ensure skills and competencies of elderly VI people to manage and take full advantage of various available technologies, mobile applications and digital services. Peers, neighbours and close ones should be posed to information on VI and its' effects on everyday lives of people suffering from it in order to ease social interaction situations between VI elderly people and their sighted peers.

Remote digital services and a digital self-service society have their downsides. VI elderly people do not necessarily have to leave their home to run errands, attend hobbies or meet other people. Modern society may displace older people who do not have access to help and support, but also empower those who adopt new technologies and services into their daily lives and to support meaningful lives. This study supports what Colenbrander and Fletcher (2018) highlight that 
fundamentally LVR is about promoting quality of life and optimal functioning in society. Individual service design is needed to achieve this goal. VI people themselves can provide valuable information on their surroundings, meaningful places and interactions regarding their living environment (Leibing et al., 2016) which raises awareness of emotional and psychosocial aspects of home environment. This information should be benefited in person-centered LVR goal-setting and in moving towards "sensory impairment -friendliness" as one aspect of accessibility in society. 


\section{Conclusion}

The study's elderly respondents appeared to be relatively active and they were satisfied with their home environments. Despite this, their experiences underline the significance of feelings of restrictiveness, reduced social participation, and the risk of social isolation resulting from VI, all of which threaten the HRQoL. To address these challenges and to support active participation of elderly individuals with VI in their environments and society, it appears necessary to extend the key ambitions and objectives of the LVR process beyond the four walls of the home. 


\section{Acknowledgments}

The authors want to express deep gratitude to the study's elderly participants for opening up their homes and living environments to the researchers.

\section{Declaration of interest statement}

The authors have no competing interests to declare. 


\section{References}

Alma MA, Van Der Mei SF, Melis-Dankers BJM, Van Tilburg TG, Groothoff JW, Suurmeijer TPBM. 2011. Participation of the elderly after vision loss. Disabil Rehabil. 1:63-72.

Barry A, Heale R, Pilon R, Lavoie AM. 2018. The meaning of home for ageing women living alone: An evolutionary concept analysis. Health Soc Care Community 26:e337344.

Binns AM, Bunce C, Dickinson C, Harper R, Tudor-Edwards R, Woodhouse M, Linck P, Suttie A, Jackson J, Lindsay J, Wolffsohn J, Hyghes L, Margrain TH. 2012. How effective is low vision service provision? A systematic review. Surv Ophthalmol. 57(1):34-65.

Bourne, R. R. A., Flaxman, S. R., Braithwaite, T., Cicinelli, M. V., Das, A., Jonas, J. B., \& Taylor, H. R. (2017). Magnitude, temporal trends, and projections of the global prevalence of blindness and distance and near vision impairment: A systematic review and meta-analysis. Lancet Glob Health. 5: 888-897.

Brundle C, Waterman HA, Ballinger C, Olleveant N, Skelton DA, Stanford P, Todd C. (2015). The causes of falls: views of older people with visual impairment. Health Expect 18, 2021-2031.

Cho HY, MacLachlan M, Clarke M, Mannan H. 2016. Accessible home environments for people with functional limitations: A systematic review. Int J Environ Res Public Health. 13:826

Colenbrander A, Fletcher DC. 2018. Vision Rehabilitation. J Neuro-Ophthalmol. 38: 135-137.

Elo S. 2006. A theory of an environment supporting the well-being of home-dwelling elderly from Northern Finland [dissertation]. Oulu: University of Oulu. [Accessed 2020 May 8] http://jultika.oulu.fi/files/isbn9514281950.pdf.

Elo S, Saarnio R, Isola A. 2011. The physical, social and symbolic environment supporting the well-being of home-dwelling elderly people. Int J Circumpolar Health. 70(1):90-100.

Elo S, Kääriäinen M, Isola A, Kyngäs H. 2013. Developing and testing a middle-range theory of the well-being supportive physical environment of home-dwelling elderly. Sci World J. Vol. 2013, Article ID 945635.

Eurostat. Population structure and ageing. 2018. [accessed 2019 May 14]

https://ec.europa.eu/eurostat/statisticsexplained/index.php/Population_structure_and_ageing

Finnish National Board on Research Integrity (TENK). 2012. [Accessed 2020 May 4] https://www.tenk.fi/en 
Futurage: A roadmap for European Aging Research. October 2011. [Accessed 2019 April 29]

http://www.foragenetwork.eu/download/database/aa0a138a5540295a9a5e4baa9d12a47 $0 /$

[IFA] International Federation on Aging. The high cost of low vision. The evidence on aging and the loss of sight. [Accessed 2019 May 14] https://www.ifa-fiv.org/wpcontent/uploads/2013/02/The-High-Cost-of-Low-Vision-The-Evidence-on-Ageing-andthe-Loss-of-Sight.pdf

Ivanoff SD, Sonn U, Lundberg-Lindqvist B, Sjöstrand J, Steen B. 2000. Disability in daily life activities and visual impairment: a population study of 85-year-old people living at home. Scand J Occup Ther. 7:148-155.

Khosravi P, Rezvani A, Wiewiora A. 2016. The impact of technology on older adults'social isolation. Comput Hum Behav. 63: 594-603.

LaGrow S, Yeung P, Towers A, Alpass, Stephens C. 2011. Determinants of the overall quality of life of older persons who have difficulty seeing: the importance of the ability to get around. J Vis Impair Blind. Oct-Nov 2011.

Leibing A, Guberman N, Wiles J. 2016. Liminal homes: Older people, loss of capacities, and the present future of living spaces. J Aging Stud. 37:10-19.

Lewis A, Torrington J. 2013. Extra-care housing for people with sight loss: Lightning and design. Lighting Res Technol. 45: 345-361.

Macnaughton J, Latham K, Vianya-Estopa M. 2019. Rehabilitation needs and activity limitations of adults with a visual impairment entering a low vision rehabilitation service in England. Ophthalmic Physiol Opt. 39: 113-126.

Markowitz SN. 2016. State-of-the-art: low vision rehabilitation. Can J Ophthalmol 51(2): 59-66.

Nygren C, Oswald F, Iwarsson S, Fänge A, Sixsmith J, Schilling O, Sixsmith A, Széman Z, Tomsone S, Wahl H-W. 2007. Relationships between objective and perceived housing in very old age. Gerontologist. 47(1):85-95.

Ojamo M. 2017. Annual statistics. The Finnish register of visual impairment. National instate for health and welfare (THL). [Accessed 2019 April 29]

https://www.nkl.fi/index.php?_file_display_id=12663

Patino CM, McKean-Cowdin R, Azen SP, Allison JC, Choudhury F, Varma R. 2010. Central and Peripheral Visual Impairment and the risk of Falls and Falls with injury. Ophthalmology. 117: 199-206.

Positive Aging 2016. National Indicators Report. Minister for Health, Dublin, Ireland. [Accessed 2018 Dec 17] https://health.gov.ie/wp-content/uploads/2016/11/NationalIndicators-Report-Single-W.pdf 
PRO HEALTH 65+ Health promotion and prevention of risk - action for seniors. Project policy brief 3, April 2016. Health Promotion interventions: effective in elderly population. [Accessed 2019 Jan 11] http://www.prohealth65plus.eu/userfiles/downloads/policybriefs/PROHealth\%2065+\%20Policy\%20brief\%203.pdf

Rantakokko M, Iwarsson S, Kauppinen M, Leinonen R, Heikkinen E, Rantanen T. 2010. Quality of life and barriers in the urban outdoor environment in old age. J Am Geriatr Soc. 58:2154-2159.

Rechel B, Grundy E, Robine J-M, Cylus J, Mackenbach JP, Knai C, McKee M. 2013. Ageing in the European Union. Published online March 27, 2013. Lancet. DOI: https://doi.org/10.1016/S0140-6736(12)62087-X

Riazi A, Boon MY, Bridge C, Dain SJ. 2012. Home modification guidelines as recommended by visually impaired people. Journal of Assistive Technologies. 6(8): 270-284.

Riazi A, Riazi F, Yoosfi R, Bahmeei F. 2016. Outdoor difficulties experienced by a group of visually impaired Iranian people. J Curr Ophthalmol. 28:85-90.

Rokicki W, Drozdzowska B, Czekajto A, Grzeszczak W, Wiktor K, Majewski W, Pluskiewicz W. 2016. Relationship between visual status and functional status and the risk of falls in women. The RAC-OST-POL study. Arch Med Sci. 12(6):1232-1238.

Schorr AV, Khalaila R. 2018. Aging in place and quality of life among the elderly in Europe: A moderated mediation model. Arch Gerontol Geriatr. 77:196-204.

Sintonen, H. 2001. The 15D instrument of health related quality of life: properties and applications. Ann Med. 33:328-336.

Sintonen H. 15D@ instrument. [Accessed 2018 Nov 27] http://www.15dinstrument.net/15d/

Steptoe A, Deaton A, Stone AA, 2015. Subjective wellbeing, health, and aging. Lancet 385(14):640-648.

Tomsone S, Horstmann V, Oswald F, Iwarsson S. 2013. Aspects of housing and perceived health among ADL independent and ADL dependent groups of older people in three national samples. Aging Clin Exp Res. 25: 317-328.

[UNECE] United Nations Economic Commission for Europe 2012. Active ageing. Policy Brief on Aging No.13. [Accessed 2020 May 4] https://www.unece.org/fileadmin/DAM/pau/age/Policy_briefs/ECE-WG.1.17.pdf

Wang D, MacMillan T. 2013. The benefits of gardening for older adults: a systematic review of the literature. Act Adapt Aging. 37: 153-181. 
Wong WL, Su X, Li X, Cheung CMG, Klein R, Cheng C-Y, Wong TY. 2014. Global prevalence of age-related macular degeneration and disease burden projection for 2020 and 2040: a systematic review and meta-analyses. Lancet Glob Health 2:e106-116.

Wood JM, Lacherez P, Black AA, Cole MH, Boon MY, Kerr GK. 2011. Risk of falls, injurious falls, and other injuries resulting from visual impairment among older adults with age-related macular degeneration. Invest Ophthalmol Vis Sci. 52(8): 5088-5092.

[WHO] World Health Organization (2003). Prevention of blindness \& deafness. Consultation on development of standards for characterization of vision loss and functioning. Geneva. [Accessed 2019 April 26]

https://apps.who.int/iris/bitstream/handle/10665/68601/WHO_PBL_03.91.pdf?sequence $=1 \&$ isAllowed $=\mathrm{y}$

[WHO] World Health Organization. 2011. World report on disability. [Accessed 2019 Jan 10] https://www.who.int/disabilities/world_report/2011/report.pdf

[WHO] World Health Organization. 2012. Strategy and action plan for healthy aging in Europe, 2012-2020.

[WHO] World Health Organization. 2013. Universal eye health: a global action plan 2014-2019. [Accessed 2019 Jan 9]

https://www.who.int/blindness/AP2014_19_English.pdf?ua=1

[WHO] World Health Organization. 2015. World report on aging and health. [Accessed 2018 Dec 17]

http://www.healthyageing.eu/sites/www.healthyageing.eu/files/resources/WHO\%20rep ort_HA2015.pdf

[WHO] World Health Organization. 2018. Age-friendly environments in Europe: indicators, monitoring and assessments. [Accessed 2019 Jan 11] http://www.euro.who.int/_data/assets/pdf_file/0003/377481/afee-indicatorsmonitoring-eng.pdf?ua $=1$

[WMA] World Medical Association. 2013. Declaration of Helsinki: Ethical principles for medical research involving human subjects. [Accessed 2019 April 26] https://www.wma.net/policies-post/wma-declaration-of-helsinki-ethical-principles-formedical-research-involving-human-subjects 